\title{
Dual-channel fluorescent probe for the simultaneous monitoring of peroxynitrite and adenosine-5'-triphosphate in cellular applications
}

Luling Wu, ${ }^{\text {ac } \ddagger}$ Jihong Liu, ${ }^{a \ddagger}$ Xue Tian, ${ }^{c \ddagger}$ Robin R. Groleau, ${ }^{c}$ Hai-Hao Han, ${ }^{d *}$ Adam C. Sedgwick, ${ }^{g}$ Han-Min Wang, ${ }^{f}$ Yang Wang, ${ }^{b}$ Yonggang Yang, ${ }^{e}$ Fang Huang, ${ }^{a}$ Steven D. Bull, ${ }^{b}$ Hua Zhang, ${ }^{\text {e* }}$ Chusen Huang ${ }^{\mathrm{b} *} \mathrm{Yi}^{\mathrm{i}}$ Zang, ${ }^{f}$ Jia Li, ${ }^{f}$ Xiao-Peng He, ${ }^{d}$ Ping Li, ${ }^{a *}$ Bo Tang, ${ }^{a}$ Tony D. James, ${ }^{\text {ace* }}$ and Jonathan L. Sesslerg*

a.College of Chemistry, Chemical Engineering and Materials Science, Key Laboratory of Molecular and Nano Probes, Ministry of Education, Collaborative Innovation Center of Functionalized Probes for Chemical Imaging in Universities of Shandong, Institutes of Biomedical Sciences, Shandong Normal University, Jinan 250014, People's Republic of China. E-mail: lip@sdnu.edu.cn

b. The Education Ministry Key Laboratory of Resource Chemistry, Shanghai Key Laboratory of Rare Earth Functional Materials, and Shanghai Municipal Education Committee Key Laboratory of Molecular Imaging Probes and Sensors, Department of Chemistry, Shanghai Normal University, 100 Guilin Road, Shanghai 200234, China. Email: huangcs@shnu.edu.cn

c.Department of Chemistry, University of Bath, Bath, BA2 7AY, UK.Email: t.d.james@bath.ac.uk

d.Key Laboratory for Advanced Materials and Joint International Research Laboratory of Precision Chemistry and Molecular Engineering, Feringa Nobel Prize Scientist Joint Research Center, School of Chemistry and Molecular Engineering, East China University of Science and Technology, 130 Meilong Rd., Shanghai 200237, China. Email: hanhaihao@126.com

e.School of Chemistry and Chemical Engineering, Henan Normal University, Xinxiang 453007, P. R. China. Email: zhanghua1106@163.com

f.National Center for Drug Screening, State Key Laboratory of Drug Research, Shanghai Institute of Materia Medica, Chinese Academy of Sciences, 189 Guo Shoujing Rd., Shanghai 201203, P. R. China.

g.Department of Chemistry, University of Texas at Austin, 105 E 24th street A5300, Austin, TX 78712-1224, USA. Email: sessler@cm.utexas.edu

${ }^{\ddagger}$ These authors contribute equally.

\section{Abstract}

The concentrations of ATP and ONOO- have been correlated with the progression a number of diseases including ischemia-reperfusion injury and drug-induced liver injury. Here, we report the development of fluorescent probe, ATP-LW, which enables the simultaneous detection of $\mathrm{ONOO}^{-}$and ATP. ONOOselectively oxidises the boronate pinacol ester of ATP-LW, to afford the fluorescent 4-hydroxy-1,8naphthalimide product NA-OH $\left(\lambda_{\mathrm{ex}}=450 \mathrm{~nm}, \lambda_{\mathrm{em}}=562 \mathrm{~nm}\right.$ or $\left.\lambda_{\mathrm{ex}}=488 \mathrm{~nm}, \lambda_{\mathrm{em}}=568 \mathrm{~nm}\right)$. While, the 
binding of ATP to ATP-LW induces the spirolactam ring opening of rhodamine to afford a highly emissive product $\left(\lambda_{\text {ex }}=520 \mathrm{~nm}, \lambda_{\mathrm{em}}=587 \mathrm{~nm}\right)$. Due to the differences in emission between the ONOO- ${ }^{-}$and ATP products, ATP-LW exhibits the unique ability to image ONOO- levels in the green channel $\left(\lambda_{\mathrm{ex}}=488 \mathrm{~nm}\right.$, $\left.\lambda_{\mathrm{em}}=500-575 \mathrm{~nm}\right)$ and ATP concentrations using the red channel $\left(\lambda_{\mathrm{ex}}=514 \mathrm{~nm}, \lambda_{\mathrm{em}}=575-650 \mathrm{~nm}\right)$. This was demonstrated using hepatocytes (HL-7702 cells) in cellular imaging experiments. The treatment of HL-7702 cell line with oligomycin A (an inhibitor of ATP synthase) resulted in a reduction of ATP in the red channel and increase in $\mathrm{ONOO}^{-}$green channel. While, the presence of SIN-1 (an exogenous ONOOdonor) results in an increase of $\mathrm{ONOO}^{-}$, and decrease in ATP. Significantly, when HL-7702 cells were treated with acetaminophen as a biological model for drug-induced liver injury, an increase in $\mathrm{ONOO}^{-}$ green and decrease in ATP red channel fluorescence was observed. These results illustrate the utility of ATP-LW as a chemical tool to simultaneously monitor ATP and ONOO- concentrations in cellular-based applications.

\section{Introduction}

Adenosine-5'-triphosphate (ATP) is known as the "molecular currency" for intracellular processes. ${ }^{1-2}$ ATP concentrations range between 1 to $10 \mathrm{mM}$, with a 1000:1 ratio between ATP and adenosine diphosphate (ADP). ${ }^{3}$ ATP aids the regulation of important cellular processes, which include cellular movement, ${ }^{4}$ neurotransmission, ${ }^{5}$ and ion channel function. ${ }^{6}$ Thus, disruption to ATP homeostasis is linked to a number of diseases, including ischemia, Parkinson's disease and hypoglycaemia. ${ }^{7}$ The cause of this disruption is often associated with oxidative stress, which involves the production of highly reactive oxygen species and reactive nitrogen species. ${ }^{8-9}$ In particular, peroxynitrite $\left(\mathrm{ONOO}^{-}\right){ }^{10}$ is a RNS that is known to inhibit ATP production by oxidatively deactivating mitochondrial ATP synthase. ${ }^{11}$ Several other studies have revealed a correlation between ATP and ONOO- $^{-}$concentrations to a range of pathological processes. ${ }^{12-14}$ Therefore, the development of a chemical tool that allows the real time monitoring of these species simultaneously in vitro and in vivo would be highly desirable.

Fluorescence imaging has emerged as an attractive technology for the real-time and noninvasive detection of biomarkers in cellular and animal-based applications. Previously, several single-analyte fluorescent probes have been reported for the selective imaging of ONOO-. ${ }^{-15-17}$ Moreover, fluorescent probes for the selective detection and visualization of ATP have been developed. ${ }^{18}$ However, to date no fluorescent probe capable of the simultaneous and independent detection and imaging of $\mathrm{ONOO}^{-}$and ATP has been reported, such a system would allow us to monitor this close relationship in real time. Here, we report the construction of ATPLW, a single fluorescent probe that enables the simultaneous detection of $\mathrm{ONOO}^{-}$and ATP. Solution data established excellent water solubility, sensitivity, and high selectivity for ONOOand ATP using their respective detection emission profiles. As a preliminary proof-of-concept 
study for cellular applications, changes in ATP and $\mathrm{ONOO}^{-}$associated with acetaminophen (APAP) were evaluated. This model was chosen due to the importance of pre-clinical tools for the screening of drug-induced liver injury (DILI) associated with drugs like APAP are crucial for drug development. ${ }^{19-21}$ Moreover, the model was selected because APAP can result in a depletion of ATP and an increase in the levels of $\mathrm{ONOO}^{-}$(Scheme S1). ${ }^{22-23}$ It is important to note, we first disclosed our probe ATP-LW as a ChemRxiv preprint, ${ }^{24}$ however, we felt that the paper could be substantially improved with the addition of biological experiments and so submission for publication of that paper was delayed. In order, to carry out those biological studies, we needed to resynthesize ATP-LW to fully evaluate the biological properties of our probe, however, the Covid-19 pandemic severely hindered our research progress. During this delay the group of Tian et al. reported a somewhat similar structure for the simultaneous detection of ATP and $\mathrm{H}_{2} \mathrm{O}_{2} .{ }^{25}$ Pointedly, Merck recognized the potential of ATP-LW and have made it commercially available. ${ }^{26}$ As such, this publication serves to fully highlight the utility of our now commercial $\mathrm{ONOO}^{-}$and ATP fluorescent probe ATP-LW. 


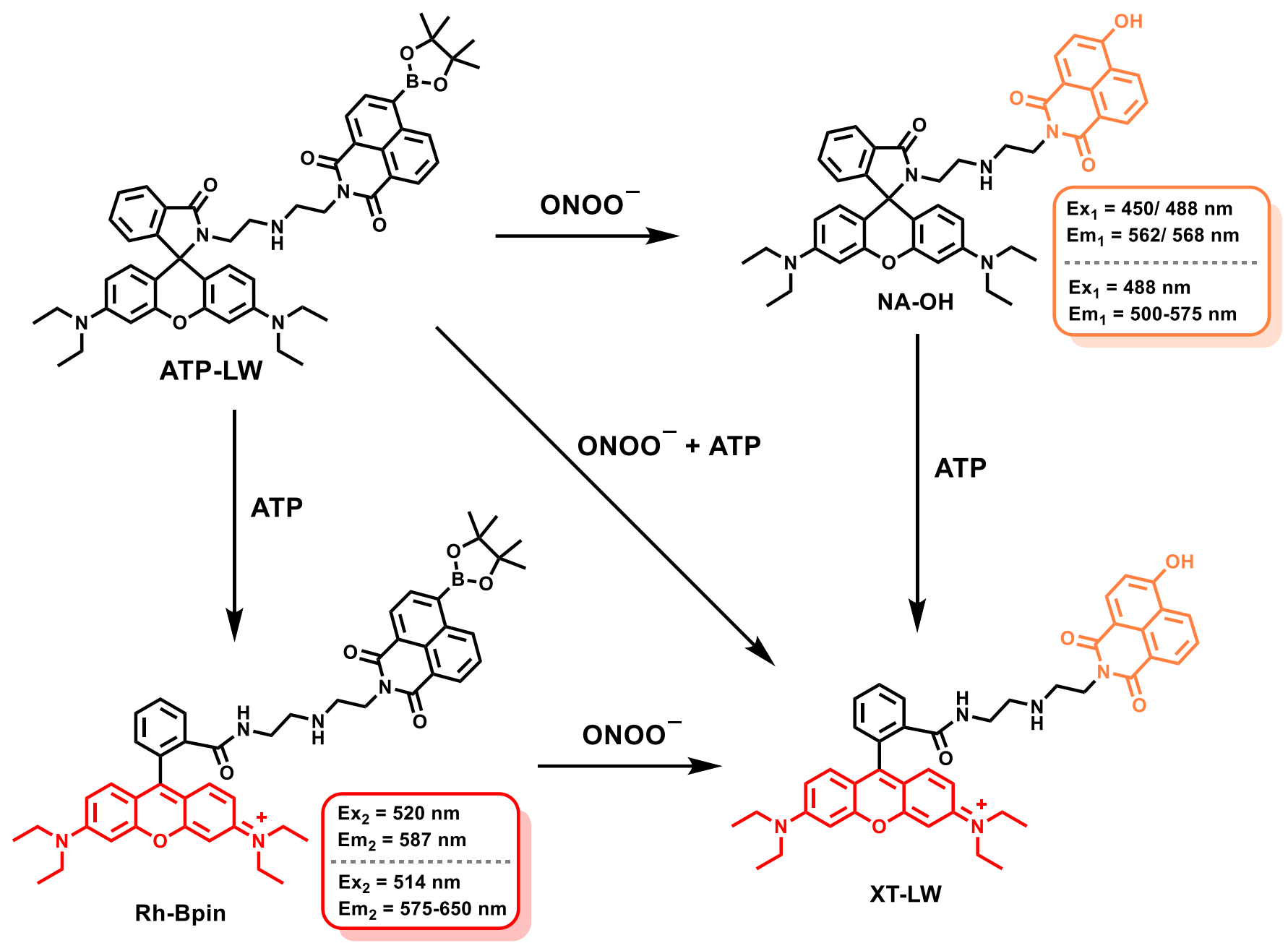

Scheme 1. Chemical Structure of ATP-LW and its fluorescence 'turn on' mechanism in the presence of $\mathrm{ONOO}^{-}$, ATP and ONOO${ }^{-}$/ATP. The addition of $\mathrm{ONOO}^{-}$leads to formation of compound NA-OH, with maximum emission at $562 \mathrm{~nm}$ excited at $450 \mathrm{~nm}$ and maximum at $568 \mathrm{~nm}$ excited at $488 \mathrm{~nm}$ in PBS buffer solution $\left(10 \mathrm{mM}, \mathrm{V} / \mathrm{V}, \mathrm{EtOH} / \mathrm{H}_{2} \mathrm{O}=1 / 99, \mathrm{pH}=7.40\right)$. The presence of ATP affords the product $\mathbf{R h}$ Bpin, with maximum emission at $587 \mathrm{~nm}$ when excited at $520 \mathrm{~nm}$ in PBS buffer solution (10 mM, V/V, $\mathrm{EtOH} / \mathrm{H}_{2} \mathrm{O}=1 / 99, \mathrm{pH}=7.40$ ).

In recent years, multi-analyte fluorescent probes have garnered attention owing to their enhanced precision for the study in question. ${ }^{27-28}$ These systems overcome the problems of using several independent fluorescent probes when they are used for understanding the relationship between more than one biological species. ${ }^{29}$ Since, the use of separate individual probes can result in erroneous analytical data due to differential permeability, distribution and reduced biocompatibility. ${ }^{29}$ Efforts from our group and others have led to the report of multianalyte systems. ${ }^{29}$ For example we have reported on dual-analyte fluorescence scaffolds for the detection of $\mathrm{ONOO}^{-}$and a second analyte. ${ }^{20,30-34}$ However, in the study of cellular processes, we note that for systems able to detect more than one analyte independently using 
different emission channels is particularly advantageous and in some cases superior to AND-logic gate systems. Since, dual-channel emission enables the evaluation of each individual species while AND logic systems only provide information on the synergy of the two species.

In this work, we rationalised a rhodamine lactam/1,8-naphthalimide hybrid structure as a scaffold for developing the dual-analyte fluorescent probe ATP-LW (Scheme 1). ${ }^{35}$ ATP-LW was synthesized over three steps (Scheme S2). The first step of the synthesis involves a Miyaura borylation reaction, which forms intermediate NA-Bpin. Diethylenetriamine was then added to a solution of rhodamine B in ethanol. This reaction afforded the desired intermediate Rh-AM as a light orange solid. The condensation reaction between NA-Bpin and Rh-AM in ethanol then afforded ATP-LW. The chemical structure of ATP-LW was fully characterized using ${ }^{1} \mathrm{H} N M R,{ }^{13} \mathrm{C}$ NMR and high-resolution mass spectrometry.

\section{Results and discussion}

Changes in the UV-Vis absorption of ATP-LW in the presence of ONOO- $^{-}$and ATP were investigated. The addition of $\mathrm{ONOO}^{-}$produced a new absorption peak at $445 \mathrm{~nm}$ which is consistent with the intramolecular charge transfer (ICT) process seen with 4-hydroxy-1,8naphthalimide product (Fig. S1). ${ }^{36}$ Similarly, the addition of ATP to ATP-LW resulted in an increase in absorption at $533 \mathrm{~nm}$. This is indicative of the ATP-induced opening of the spirolactam ring found on the rhodamine fluorophore (Fig. S2). ${ }^{18}$ ATP-LW was initially non fluorescent, due to the quenching effects of the boronic ester on the 1,8-naphthalimide unit and ring-closed rhodamine being non-fluorescent. Upon the addition of $\mathrm{ONOO}^{-}(0-16 \mu \mathrm{M}), \mathrm{a}$ significant fluorescence increase at $562 \mathrm{~nm} / 568 \mathrm{~nm}$ was observed when excited at 450/488 $\mathrm{nm}$ (Fig. 1a, Fig. S6-S8). $488 \mathrm{~nm}$ excitation wavelength was used for measurements in aqueous solution since it was the closest available excitation wavelength for cellular imaging experiments (green channel, $\lambda_{\mathrm{em}}=500-575 \mathrm{~nm}, \lambda_{\mathrm{ex}}=488 \mathrm{~nm}$ ). Minimal fluorescence increase was observed for light excitation at $520 \mathrm{~nm}$ upon the addition of $\mathrm{ONOO}^{-}(0-16 \mu \mathrm{M})$ (Fig. 1b).

The 4-hydroxy-1,8-naphthalimide product on reaction of ATP-LW with ONOO- was confirmed by LC-MS analysis. (Scheme S3, Fig. S17, and S18). Subsequently we evaluated the ability of ATP-LW to detect ATP, a large increase in fluorescence intensity at $587 \mathrm{~nm}$ (> 80-fold, see Fig. 2a and Fig. S4) was observed following the addition of ATP (0-15 mM) when excited at $520 \mathrm{~nm}$. However, the addition of ATP (0-15 $\mathrm{mM})$ to ATP-LW resulted in only a small increase in fluorescence intensity with excitation at 450 (Fig. 2b) or $488 \mathrm{~nm}$ (Fig. S5). Thus, no ATP-mediated increase using excitation at 450 and $488 \mathrm{~nm}$ was observed. This confirms the ability of ATP-LW to separately detect $\mathrm{ONOO}^{-}$and ATP in the respective excitation profiles. 
a)

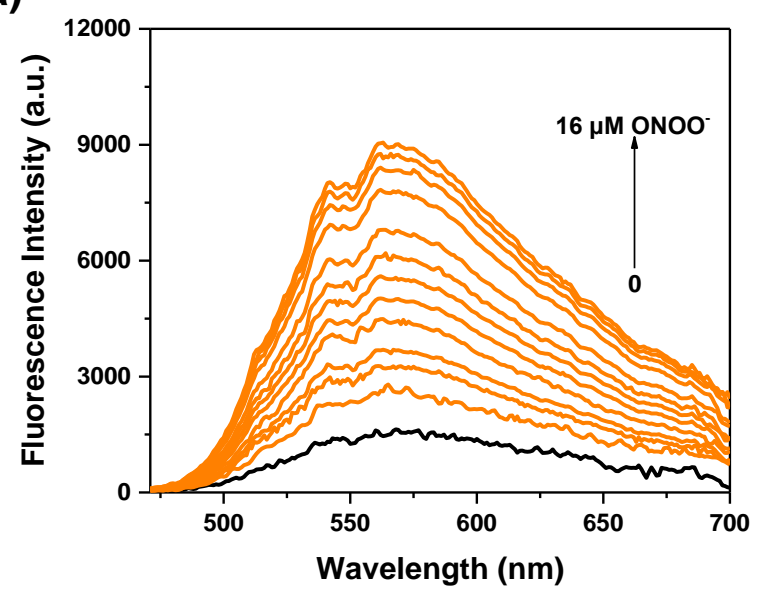

b)

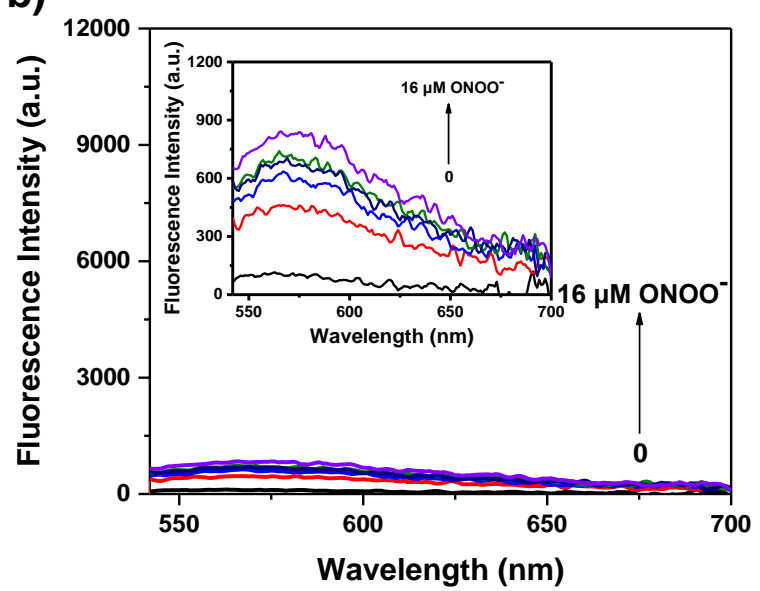

Figure 1. Changes in fluorescence emission intensity of ATP-LW $(15 \mu \mathrm{M})$ with increasing addition of $\mathrm{ONOO}^{-}$(from 0 to $16 \mu \mathrm{M}$ ) in PBS buffer solution ( $10 \mathrm{mM}, \mathrm{V} / \mathrm{V}, \mathrm{EtOH} / \mathrm{H}_{2} \mathrm{O}=1 / 99, \mathrm{pH}=7.40$ ) after $1 \mathrm{~min}$. (a) Excited at 450 (bandwidth 8) nm, (b) excited at 520 (bandwidth 8) nm. Inset: the enlarged spectra.

a)

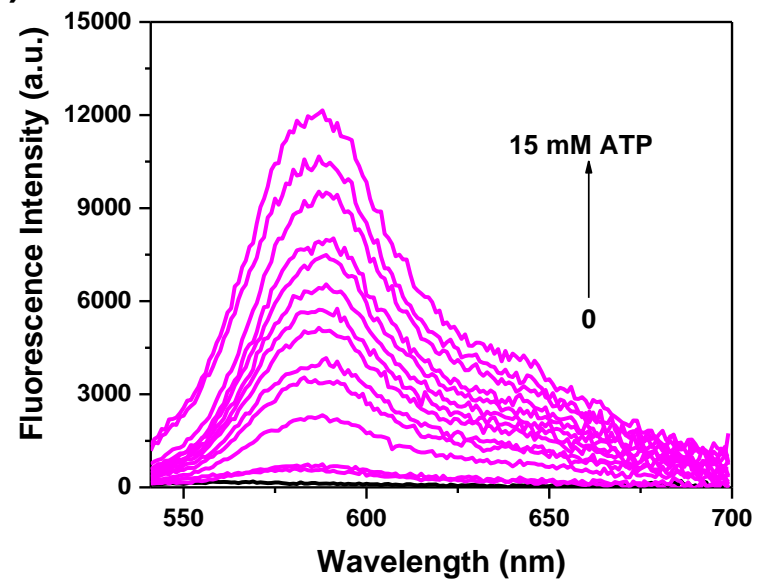

b)

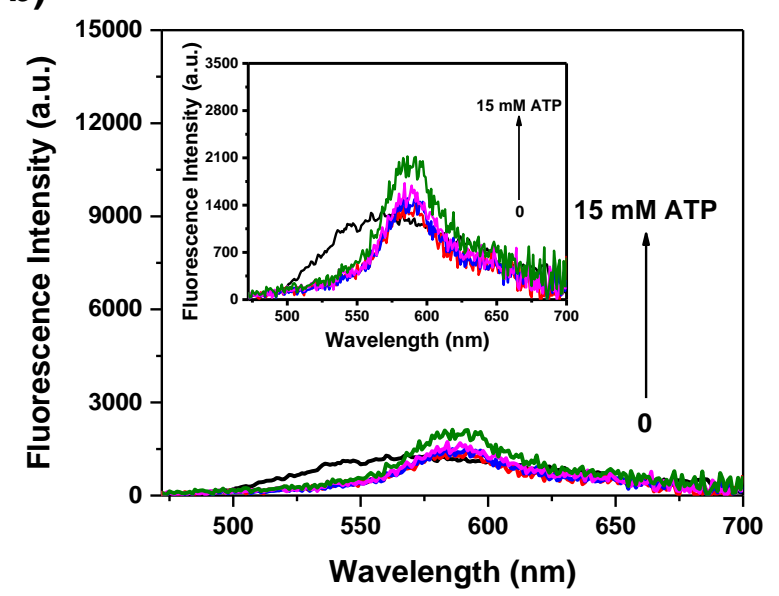

Figure 2. Changes in fluorescence emission intensity of ATP-LW $(15 \mu \mathrm{M})$ with increasing additions of ATP (from 0 to $15 \mathrm{mM}$ ) in PBS buffer solution (10 mM, V/V, EtOH/H2O = 1/99, pH = 7.40) after $100 \mathrm{~min}$. (a) Excited at 520 (bandwidth 8) nm, (b) excited at 450 (bandwidth 8) nm. Inset: enlarged spectra.

We then explored the fluorescence response of ATP-LW in the presence of both ONOO- $^{-}$and ATP. The peak at $589 \mathrm{~nm}$ for ATP-LW retained sensitivity to changes in concentration of ATP even after addition of $\mathrm{ONOO}^{-}(16 \mu \mathrm{M})$ using excitation at $520 \mathrm{~nm}$ (Fig. S11). The emission peak at 562 $\mathrm{nm}$ (Fig. S12) or $569 \mathrm{~nm}$ (Fig. S13) changed with the addition of $\mathrm{ONOO}^{-}$and was only slightly influenced by the addition of ATP (15 $\mathrm{mM})$. Importantly, since the two emission peaks are close it becomes difficult to infer whether FRET is occurring between the naphthalimide and 
rhodamine, ${ }^{35}$ as such we used density functional theoretical methods to evaluate the system which confirmed our belief that FRET was not occurring significantly between the two fluorophores within ATP-LW (Fig. S16). These results confirmed that ATP-LW could be used to detect $\mathrm{ONOO}^{-}$and ATP using two different excitation wavelengths independently (Scheme 1).

We then evaluated the selectivity of probe ATP-LW towards different biologically relevant interfering species (Fig. 3 and 4). Other ROS such as $\mathrm{H}_{2} \mathrm{O}_{2}$ and $\mathrm{HOCl}$ led to no change in fluorescence intensity of ATP-LW (Fig. 3). However, ADP (10 mM) induced fluorescence enhancements (Fig. 4). Nevertheless, this will not prevent the use of ATP-LW for the detection ATP in cells, since the concentration of ATP is around 1000-fold higher than ADP in cells. ${ }^{3}$ We then determined that ATP-LW exhibits good stability over a pH range from 3-11 (Fig. S9 and S10). The fluorescence emission of the solution formed by reaction of ATP-LW with ONOO- decreased at lower $\mathrm{pH}$, which could be ascribed to the decomposition of $\mathrm{ONOO}^{-}$in acidic solutions (Fig. S9). ${ }^{37}$ While, the fluorescence intensity at $587 \mathrm{~nm}$ decreased significantly at higher $\mathrm{pH}(\mathrm{pH}>8)$, which is most likely due to hydrolysis of ATP (Fig. S10). ${ }^{38}$ The presence of ONOO- resulted in an instantaneous and significant fluorescence increase (Fig. S14), whilst the reaction of ATP-LW and ATP was complete within 100 min (Fig. S15).

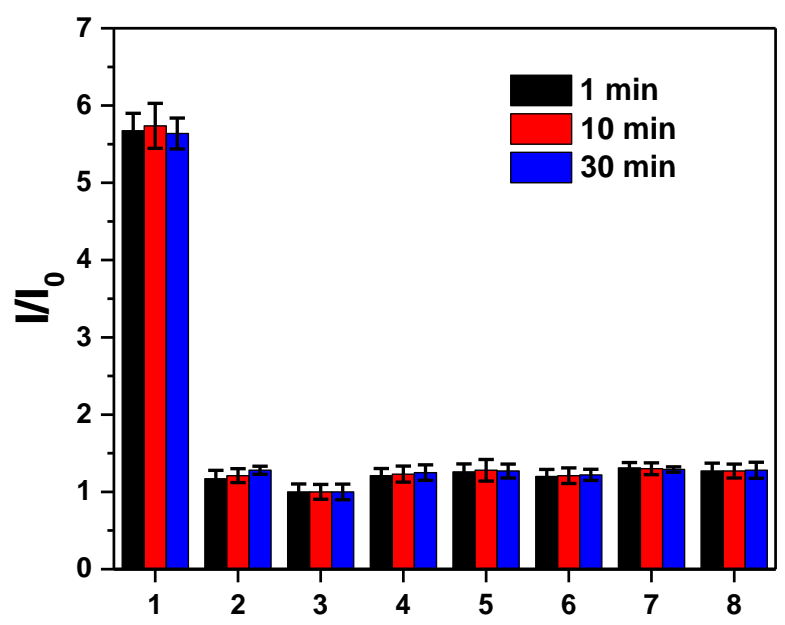

Figure 3. Selectivity bar chart for probe ATP-LW $(15 \mu \mathrm{M})$ in PBS buffer solution $\left(10 \mathrm{mM}, \mathrm{V} / \mathrm{V}, \mathrm{EtOH} / \mathrm{H}_{2} \mathrm{O}=\right.$ $1 / 99, \mathrm{pH}=7.40)$ with $\mathrm{ONOO}^{-}(16 \mu \mathrm{M})$ and other $\mathrm{ROS}(100 \mu \mathrm{M}) .1, \mathrm{ONOO}^{-} ; 2, \mathrm{H}_{2} \mathrm{O}_{2} ; 3$, probe only; $4, \mathrm{HOCl}$; $5, \mathrm{ROO}^{\bullet} ; 6,{ }^{\bullet} \mathrm{OH} ; 7, \mathrm{O}_{2}{ }^{--} ; 8,{ }^{1} \mathrm{O}_{2} ; \lambda_{\mathrm{ex} / \mathrm{em}}=450$ (bandwidth 8 ) $\mathrm{nm} / 562 \mathrm{~nm}$. Time points were taken at $1 \mathrm{~min}$ (black bars), $10 \mathrm{~min}$ (red bars) and $30 \mathrm{~min}$ (blue bars). 


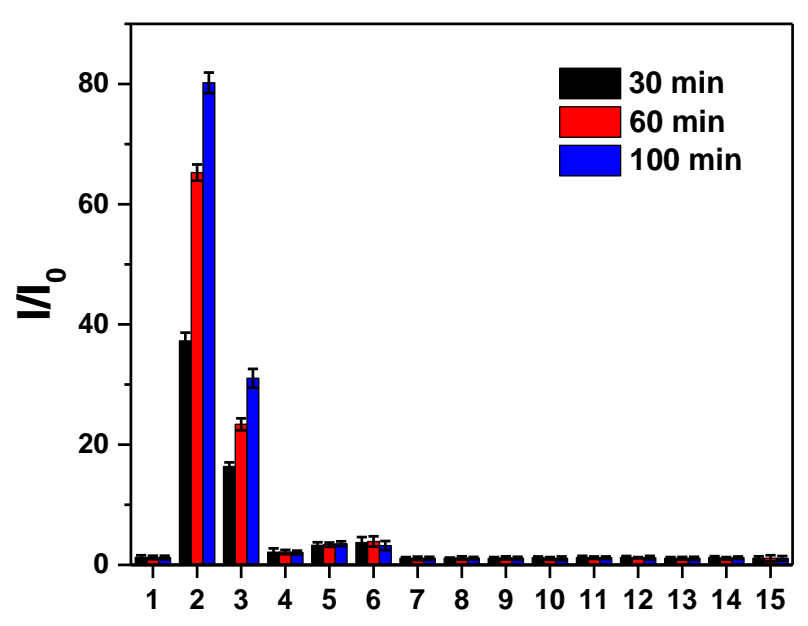

Figure 4. Selectivity bar chart of ATP-LW $(15 \mu \mathrm{M})$ in PBS buffer solution $\left(10 \mathrm{mM}, \mathrm{V} / \mathrm{V}, \mathrm{EtOH} / \mathrm{H}_{2} \mathrm{O}=1 / 99\right.$, $\mathrm{pH}=7.40)$ with ATP $(15 \mathrm{mM})$ and other interfering species. (1) probe only; (2) ATP (15 mM); (3) adenosine diphosphate (ADP, $10 \mathrm{mM}$ ); (4) uridine $5^{\prime}$-triphosphate, trisodium salt (UTP trisodium salt 10 $\mathrm{mM}$ ); (5) guanosine 5'-triphosphate, disodium salt (GTP disodium salt, $10 \mathrm{mM}$ ); (6) cytidine 5'triphosphate (CTP disodium salt, disodium salt, $10 \mathrm{mM}$ ); (7) cysteine (1 mM); (8) glutathione (1 mM); (9) hemocysteine (1 mM); (10) KCl (6 mM); (11) $\mathrm{CaCl}_{2}$ (2 mM); (12) $\mathrm{MgCl}_{2}(1 \mathrm{mM}) ;(13) \mathrm{CuCl}_{2}(100 \mu \mathrm{M}) ;(14)$ $\mathrm{ZnCl}_{2}(100 \mu \mathrm{M}) ;(15) \mathrm{NaCl}(100 \mathrm{mM}) . \lambda_{\mathrm{ex} / \mathrm{em}}=520$ (bandwidth 8$) \mathrm{nm} / 587 \mathrm{~nm}$. Time points were taken at 30 min (black bars), $60 \mathrm{~min}$ (red bars) and $100 \mathrm{~min}$ (blue bars).

These promising results in aqueous solution prompted us to explore the use of ATP-LW for imaging live cells. Firstly, we confirmed using an MTT assay that ATP-LW was non-toxic to HL7702 cells over concentrations ranging from 0 to $1 \mathrm{mM}$ (Fig. S20). The ability of ATP-LW to image $\mathrm{ONOO}^{-}$and ATP in living cells was then evaluated using excitation wavelengths of $488 \mathrm{~nm}$ and $514 \mathrm{~nm}$, respectively. One-photon imaging was then performed to evaluate the effect of ATP and ONOO- $^{-}$changes stimulated by SIN-1. ATP-LW demonstrated a clear "turn on" response with the addition of SIN-1 in the green channel (Fig. 5a and 5b). Meanwhile, a 0.55-fold decrease in red channel was observed, when compared to the control group (Fig. 5c). No interference for both green channel excited by $514 \mathrm{~nm}$ and red channel under excitation at $488 \mathrm{~nm}$ were observed for ATP-LW when HL-7702 cells were exposed to ATP and SIN-1 (a donor of ONOO-) ${ }^{39}$ simultaneously (Fig. S21). As shown in Fig. S21, there was almost no fluorescence in Channel 3 $\left(\lambda_{\mathrm{ex}}=488 \mathrm{~nm}, \lambda_{\mathrm{em}}=575-650 \mathrm{~nm}\right)$ as well as weak fluorescence in Channel $4\left(\lambda_{\mathrm{ex}}=514 \mathrm{~nm}, \lambda_{\mathrm{em}}=\right.$ 520-575 nm). The two fluorescence channels affected by SIN-1 were then evaluated using the ONOO- $^{-}$scavenger, uric acid. ${ }^{40}$ The addition of uric acid $(500 \mu \mathrm{M})$ and SIN-1 $(1.0 \mathrm{mM})$ led to a 0.35 -fold decrease in the average green fluorescence intensity and 1.83-fold enhancement in the average red fluorescence intensity, when compared with the corresponding SIN-1 group (green 
and red channels, respectively, Fig. 5). These results indicate that an increase of $\mathrm{ONOO}^{-}$can result in depletion of ATP, which is in agreement with previous studies. ${ }^{22}$

We then set out to evaluate how imbalances in the energy metabolism instigated by obstructing ATP production can influence production of $\mathrm{ONOO}^{-}$. Oligomycin A inhibits ATP synthase by blocking its Fo unit. ${ }^{41}$ As shown in Fig. 6, after the hepatocytes were incubated with oligomycin A $(25 \mu \mathrm{M})$ for $1 \mathrm{~h}$, the intensities obtained from imaging in the red channel revealed a significant decrease (35\% of the initial level, Fig. $6 \mathrm{c}$ ), and 1.55-fold increase in the green channel suggestive of an increase in level of $\mathrm{ONOO}^{-}$when compared with healthy hepatocytes (Fig. 6b). Next, following the addition of exogenous ATP $(10 \mathrm{mM})$, the intensity of green channel is $85 \%$ of the control group (Fig. 6b) and the intensity from the red channel increased to almost the same level as the control group (Fig. 6c), which indicated that the addition of ATP induced recovery. These results indicated production of ROS/RNS is affected by mitochondrial damage to ATP synthesis, leading to an increase of $\mathrm{ONOO}^{-}$. While this influence could be diminished by the addition of ATP. ${ }^{25}$ Which confirms that the inhibition of ATP synthase results in the production of $\mathrm{ONOO}^{-}$.

ROS and RNS are regarded as biomarkers in DILI, which has been detected by various fluorescent probes. ${ }^{19,} 21$ Previous studies confirm ATP production is decreased via APAP. ${ }^{22,42-43}$ APAP-induced hepatoxicity was chosen as the model for ATP-LW to investigate whether the two species can be regarded as early diagnostic biomarkers. Treatment of HL-7702 cells with APAP produced a marked increase in fluorescence in the green channel and significant decrease in fluorescence in the red channel (Fig. 7), indicating upregulation of intracellular ONOO- $^{-}$and depletion of ATP after administration of APAP, and illustrating the ability of our probe to detect concentration changes of the two biomarkers by one-photon confocal imaging in DILI. This was further confirmed using NAC, which can increase cellular concentrations of GSH. ${ }^{44-45} \mathrm{GSH}$ is capable of eliminating $\mathrm{ONOO}^{-}$and as such has been used to help treat an APAP overdose. ${ }^{46-47}$ Therefore, as expected upon addition of NAC, the fluorescence intensity in the green channel decreased and red channel increased (Fig. 7).

Our attention then turned to two-photon imaging ${ }^{48}$ of ONOO- $^{-}$and ATP using ATP-LW, with the same cell models used above. The results (Fig. 8) confirm the ability of ATP-LW to image both ONOO- $^{-}$and ATP, using an excitation of $976 \mathrm{~nm}$ for the former, and $1028 \mathrm{~nm}$ for the latter. Since the power for excitation at $1028 \mathrm{~nm}$ for a two-photon microscope is lower, we extended the emission range for the red channel (i.e. 575-680 $\mathrm{nm}$ ). 


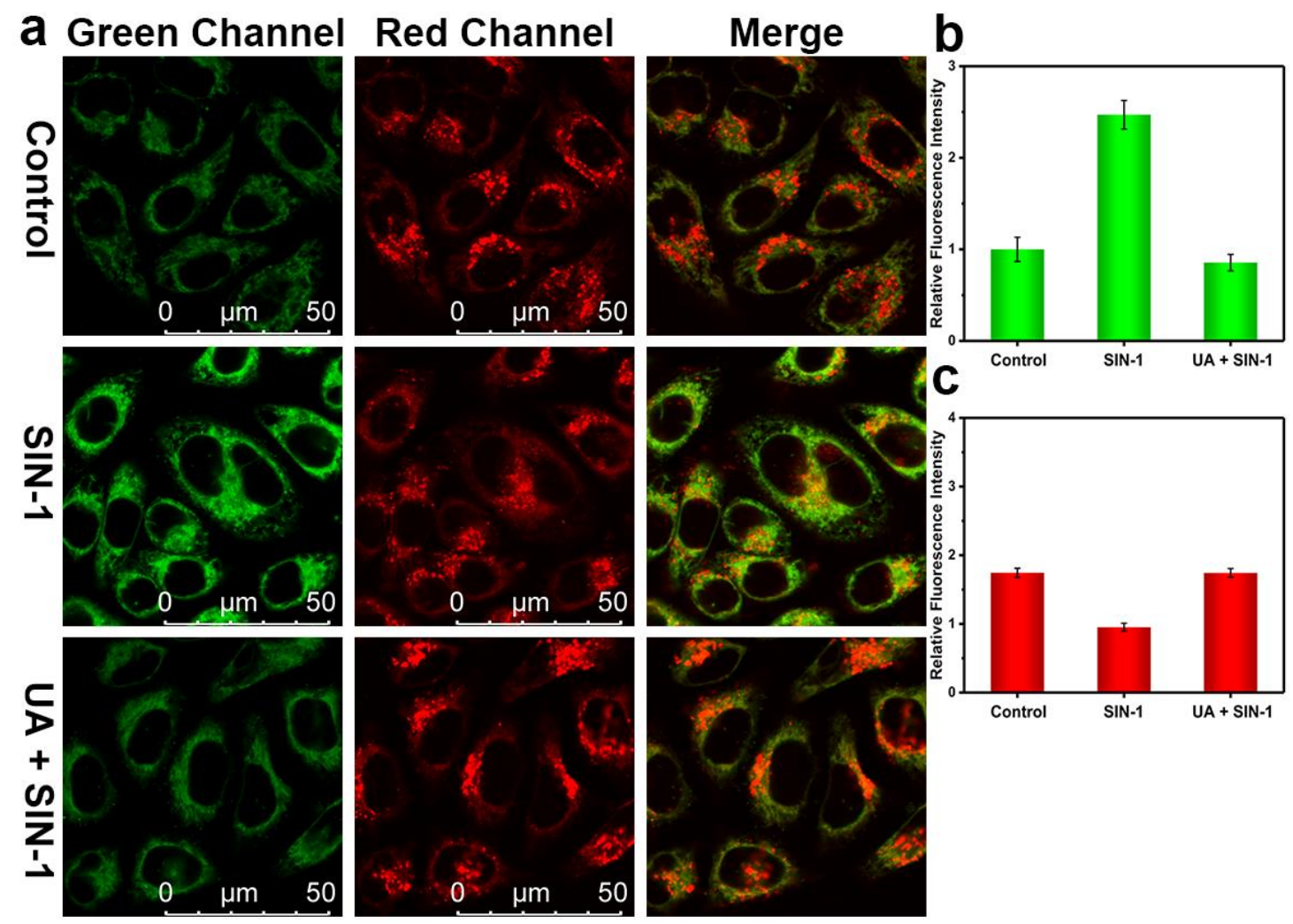

Figure 5. One-photon confocal imaging of $\mathrm{ONOO}^{-}$and ATP levels in hepatocytes treated with SIN-1 or SIN-1/uric acid. (a) One-photon fluorescence images of HL-7702 cells with the addition of SIN-1 (1 mM, 1 h) and uric acid $(500 \mu \mathrm{M}, 1 \mathrm{~h})$ for green $\left(\mathrm{ONOO}^{-}\right)$and red (ATP) channels. Control group: cells were stained with probe ATP-LW $(20 \mu \mathrm{M})$ for $20 \mathrm{~min}$. SIN-1 group: cells were incubated with SIN-1 (1 mM) for 1 $\mathrm{h}$, then stained with probe ATP-LW $(20 \mu \mathrm{M})$ for $20 \mathrm{~min}$. UA + SIN-1 group: Cells were pretreated with uric acid $(500 \mu \mathrm{M})$ for $1 \mathrm{~h}$ and followed by adding SIN-1 $(1 \mathrm{mM})$ for $1 \mathrm{~h}$ and then stained with probe ATP-LW $(20 \mu \mathrm{M})$ for $20 \mathrm{~min}$. Green fluorescence channel for $\mathrm{ONOO}^{-}: \lambda_{\mathrm{ex}}=488 \mathrm{~nm}, \lambda_{\mathrm{em}}=500-575 \mathrm{~nm}$. Red fluorescence channel for ATP: $\lambda_{\mathrm{ex}}=514 \mathrm{~nm}, \lambda_{\mathrm{em}}=575-650 \mathrm{~nm}$. (b) Green relative fluorescence intensity output of three groups. (c) Red relative fluorescence intensity output of three groups. Note: The green fluorescence intensity of the control group is defined as 1.0. The data are expressed as the mean \pm SD. Similar results were obtained from-five independent experiments. 


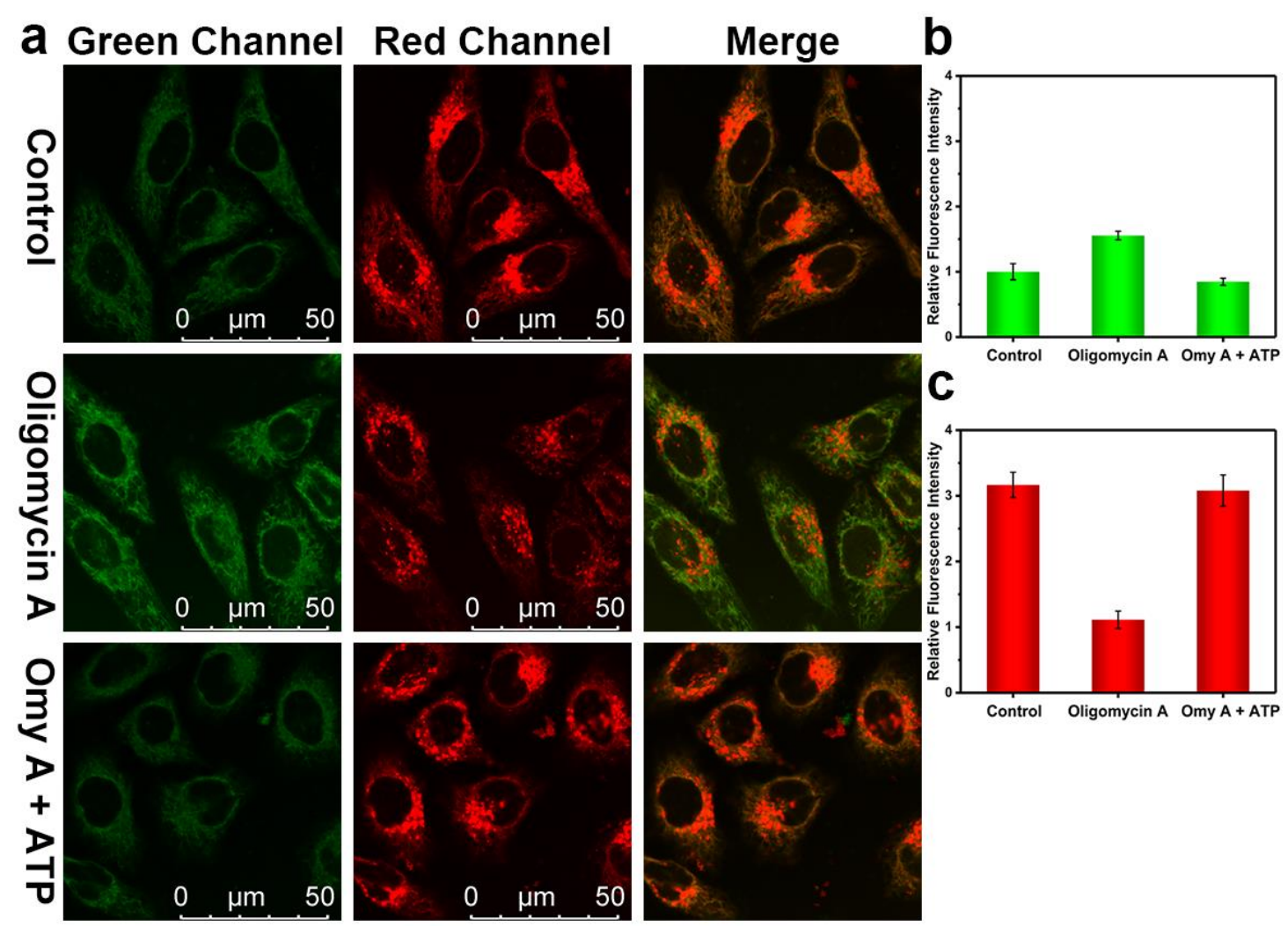

Figure 6. One-photon confocal imaging of $\mathrm{ONOO}^{-}$and ATP levels in hepatocytes. (a) One-photon fluorescence images of HL-7702 cells with the addition of oligomycin A ( $25 \mu \mathrm{M}, 1 \mathrm{~h}$ ) and ATP (10 mM, $1 \mathrm{~h}$ ) for green $\left(\mathrm{ONOO}^{-}\right)$and red (ATP) channels. Control group: Cells were stained with probe ATP-LW $(20 \mu \mathrm{M})$ for 20 min. Oligomycin group: Cells were incubated with oligomycin $A(25 \mu M)$ for $1 \mathrm{~h}$, then stained with probe ATP-LW $(20 \mu \mathrm{M})$ for $20 \mathrm{~min}$. Oligomycin A + ATP group: Cells were pretreated with oligomycin A $(25 \mu \mathrm{M})$ for $1 \mathrm{~h}$ and followed by adding ATP $(10 \mathrm{mM})$ for $1 \mathrm{~h}$ and then stained with probe ATP-LW $(20 \mu \mathrm{M})$ for $20 \mathrm{~min}$. Green fluorescence channel for ONOO${ }^{-}: \lambda_{\mathrm{ex}}=488 \mathrm{~nm}, \lambda_{\mathrm{em}}=500-575 \mathrm{~nm}$. Red fluorescence channel for ATP: $\lambda_{\mathrm{ex}}=514 \mathrm{~nm}, \lambda_{\mathrm{em}}=575-650 \mathrm{~nm}$. (b) Green relative fluorescence intensity output of three groups. (c) Red relative fluorescence intensity output of three groups. Note: The green fluorescence intensity of the control group is defined as 1.0. The data are expressed as the mean \pm SD. Similar results were obtained from five independent experiments. 


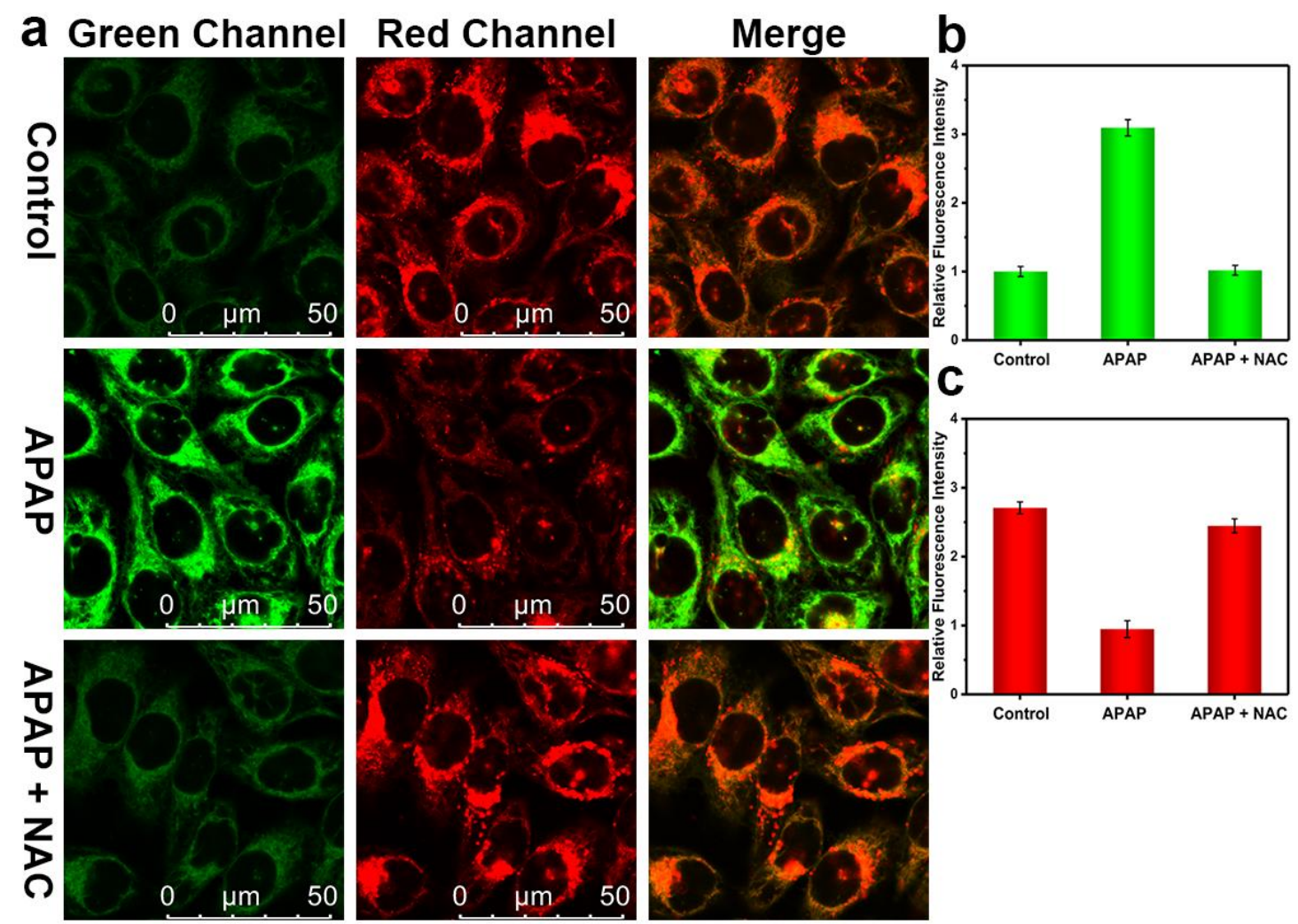

Figure 7. One-photon confocal images of APAP-induced injury and its remediation by NAC in HL-7702 cells. (a) One-photon fluorescence images of HL-7702 cells with the addition of APAP ( $20 \mathrm{mM}, 2 \mathrm{~h}$ ) and NAC ( $2 \mathrm{mM}, 2 \mathrm{~h}$ ) for green $\left(\mathrm{ONOO}^{-}\right)$and red (ATP) channels. Control group: Cells were stained with probe ATP-LW $(20 \mu \mathrm{M})$ for $20 \mathrm{~min}$. APAP group: Cells were incubated with APAP $(20 \mathrm{mM})$ for $2 \mathrm{~h}$, and then stained with probe ATP-LW $(20 \mu \mathrm{M})$ for $20 \mathrm{~min}$. APAP + NAC group: Cells were pretreated with NAC ( 2 $\mathrm{mM}$ ) for $2 \mathrm{~h}$ and then incubated with APAP $(20 \mathrm{mM})$ for $2 \mathrm{~h}$, followed by staining with probe ATP-LW (20 $\mu \mathrm{M})$ for another $20 \mathrm{~min}$. Green fluorescence channel for $\mathrm{ONOO}^{-}: \lambda_{\mathrm{ex}}=488 \mathrm{~nm}, \lambda_{\mathrm{em}}=500-575 \mathrm{~nm}$. Red fluorescence channel for ATP: $\lambda_{\mathrm{ex}}=514 \mathrm{~nm}, \lambda_{\mathrm{em}}=575-650 \mathrm{~nm}$. (b) Green relative fluorescence intensity output of three groups. (c) Red relative fluorescence intensity output of three groups. Note: The green fluorescence intensity of the control group is defined as 1.0. The data are expressed as the mean \pm SD. Similar results were obtained from five independent experiments. 


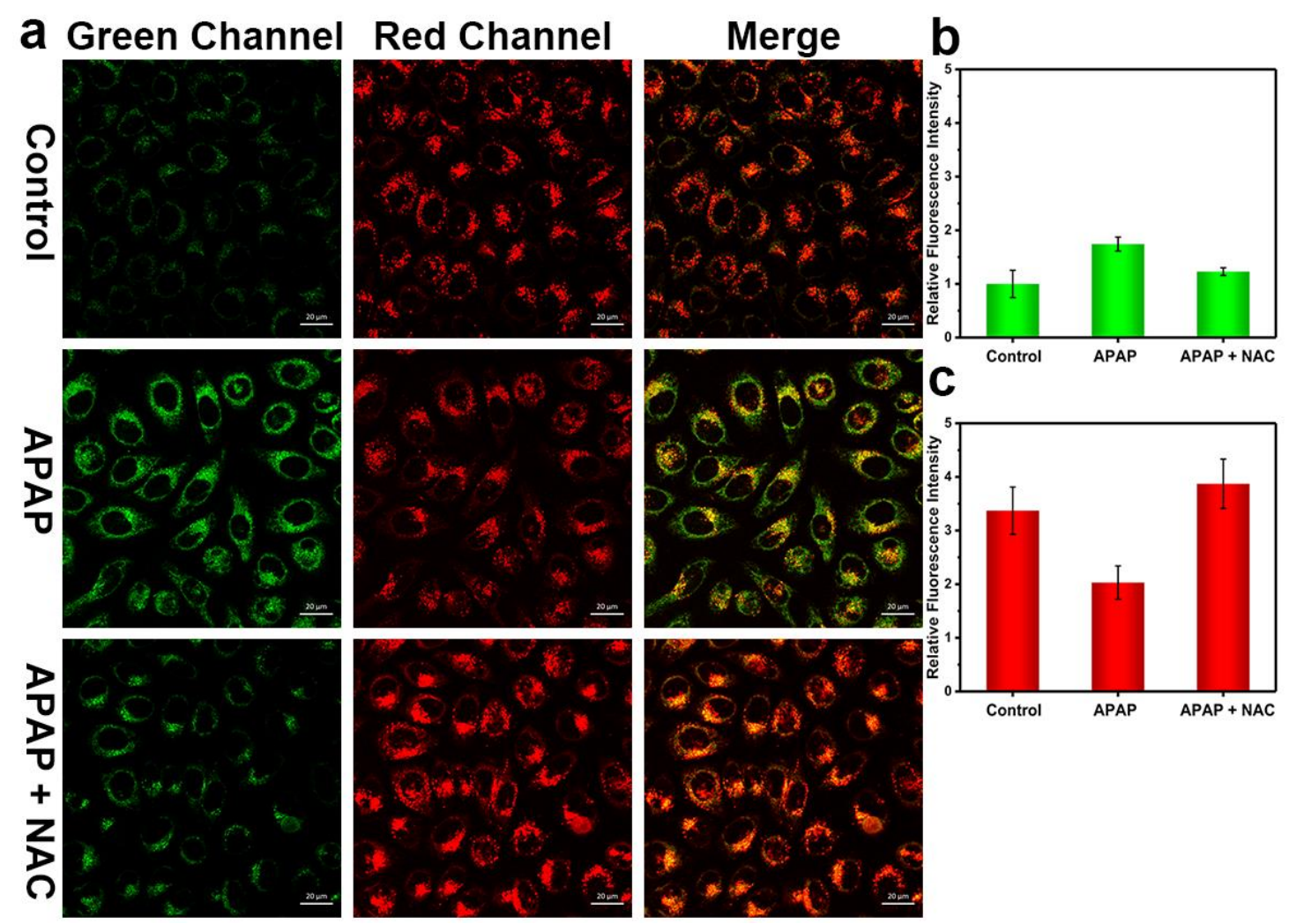

Figure 8. Two-photon fluorescence images of APAP-induced injury with HL-7702 cells. (a) Two-photon fluorescence images of HL-7702 cells with the addition of APAP $(20 \mathrm{mM}, 2 \mathrm{~h})$ and NAC $(2 \mathrm{mM}, 2 \mathrm{~h})$ for green $\left(\mathrm{ONOO}^{-}\right)$and red (ATP) channels. Control group: Cells were stained with probe ATP-LW $(20 \mu \mathrm{M})$ for 20 min. APAP group: Cells were incubated with APAP $(20 \mathrm{mM})$ for $2 \mathrm{~h}$, and then stained with probe ATPLW $(20 \mu \mathrm{M})$ for $20 \mathrm{~min}$. APAP + NAC group: Cells were pretreated with NAC $(2 \mathrm{mM})$ for $2 \mathrm{~h}$ and then incubated with APAP $(20 \mathrm{mM})$ for $2 \mathrm{~h}$, followed by staining with probe ATP-LW $(20 \mu \mathrm{M})$ for another 20 min. Two-photon green fluorescence channel for $\mathrm{ONOO}^{-}: \lambda_{\mathrm{ex}}=976 \mathrm{~nm}, \lambda_{\mathrm{em}}=500-575 \mathrm{~nm}$. Two-photon red fluorescence channel for ATP: $\lambda_{\mathrm{ex}}=1028 \mathrm{~nm}, \lambda_{\mathrm{em}}=575-680 \mathrm{~nm}$. (b) Green relative fluorescence intensity output of three groups. (c) Red relative fluorescence intensity output of three groups. Note: The green fluorescence intensity of the control group is defined as 1.0. The data are expressed as the mean \pm SD. Similar results were obtained from five independent experiments.

In summary, we have developed a novel dual-analyte fluorescent probe (ATP-LW), which exhibited fluorescence responses towards $\mathrm{ONOO}^{-}$and ATP simultaneously using different excitation wavelengths. The design strategy for ATP-LW combines two responsive units towards two biomarkers, respectively. ${ }^{29}$ Upon sole addition of $\mathrm{ONOO}^{-}$, the 4-hydroxy-1,8-naphthalimide luminesces $\left(\lambda_{\mathrm{ex}}=450 \mathrm{~nm}, \lambda_{\mathrm{em}}=562 \mathrm{~nm}\right.$ or $\lambda_{\mathrm{ex}}=488 \mathrm{~nm}, \lambda_{\mathrm{em}}=568 \mathrm{~nm}$ ); when only ATP is present, the ring-opening rhodamine luminesces $\left(\lambda_{\mathrm{ex}}=520 \mathrm{~nm}, \lambda_{\mathrm{em}}=587 \mathrm{~nm}\right)$. In order to detect ONOOand ATP with minimal cross-talk, we choose emission profiles matching Fig S11 and S12 (i.e. green chanenel: $\lambda_{\mathrm{ex}}=488 \mathrm{~nm}, \lambda_{\mathrm{em}}=500-575 \mathrm{~nm}$; red channel: $\lambda_{\mathrm{ex}}=514 \mathrm{~nm}, \lambda_{\mathrm{em}}=575-650 \mathrm{~nm}$ ) 
for cellular imaging. Importantly, using ATP-LW we have been able (using two different channels) to monitor in real-time for the first time the enhancement of $\mathrm{ONOO}^{-}$and depletion of ATP during APAP-induced hepatotoxicity. Moreover, we have illustrated the signaling pathways for APAP-induced toxicity where $\mathrm{ONOO}^{-}$increase and ATP depletion are responsible for hepatic necrosis. ${ }^{49}$ We anticipate that ATP-LW can be extended for imaging the important fluctuations of these two biomarkers in other diseases such as ischemia-reperfusion injury. ${ }^{14}$

\section{Acknowledgements}

L.W. wishes to thank China Scholarship Council and the University of Bath for supporting his PhD in the UK. R. R. G. wishes to thank the EPSRC Centre for Doctoral Training in Catalysis (EP/L016443/1). We would like to thank the EPSRC and the University of Bath for funding. T. D. J. wishes to thank the Royal Society for a Wolfson Research Merit Award and the Open Research Fund of the School of Chemistry and Chemical Engineering, Henan Normal University for support (2020ZD01). P. L. and B. T. thank the National Natural Science Foundation of China (21927811, 22074083), the Key Research and Development Program of Shandong Province (2018YFJH0502), and the National Science Foundation of Shandong Province of China (ZR2020ZD17). C. H. thanks National Natural Science Foundation of China (Grants 21672150, 21302125), Alexander von Humboldt Foundation (AvH), Shanghai Rising-Star Program (19QA1406400), Shanghai Government (18DZ2254200). H.-H. H. would like to thank the Project funded by China Postdoctoral Science Foundation (No. 2020M681196). X.-P. H. thank the National Natural Science Foundation (No. 91853201), the Shanghai Municipal Science and Technology Major Project (2018SHZDZX03), the National Key Sci-Tech Special Projects of Infection Diseases of China (2018ZX10732202) and the International Cooperation Program of Shanghai Science and Technology Committee (17520750100). H. Z. thanks the National Natural Science Foundation of China (21722501, 11974103), the zhongyuan high level talents special support plan-Science and Technology Innovation Leading Talents (204200510006), and Program for Science Technology Innovation Talents in Universities of Henan Province (21HASTIT019). J. L. S. thanks the Robert A. Welch Foundation (F-0018) for support. NMR characterisation facilities were provided by the Material and Chemical Characterisation Facility $\left(\mathrm{MC}^{2}\right)$ at the University of Bath (http://go.bath.ac.uk/mc2), and we would like to thank $\mathrm{Dr}$ John Lowe for his support with characterization. The EPSRC UK National Mass Spectrometry Facility at Swansea University is thanked for analyses. All data supporting this study are provided as supplementary information accompanying this paper (ESI + ). 


\section{Conflicts of interest}

\section{No conflicts of interest}

\section{Notes and references}

1. Knowles, J. R., Enzyme-catalyzed phosphoryl transfer reactions. Annu. Rev. Biochem. 1980, 49, 877-919.

2. Higgins, C. F.; Hiles, I. D.; Salmond, G. P. C.; Gill, D. R.; Downie, J. A.; Evans, I. J.; Holland, I. B.; Gray, L.; Buckel, S. D.; Bell, A. W.; Hermodson, M. A., A family of related ATP-binding subunits coupled to many distinct biological processes in bacteria. Nature 1986, 323, 448-450.

3. Zimmerman, J. J.; von Saint André-von Arnim, A.; McLaughlin, J., Chapter 74 - Cellular Respiration. In Pediatric Critical Care (Fourth Edition), Fuhrman, B. P.; Zimmerman, J. J., Eds. Mosby: 2011; pp 1058-1072.

4. Davalos, D.; Grutzendler, J.; Yang, G.; Kim, J. V.; Zuo, Y.; Jung, S.; Littman, D. R.; Dustin, M. L.; Gan, W.-B., ATP mediates rapid microglial response to local brain injury in vivo. Nat. Neurosci. 2005, 8, 752-758.

5. Burnstock, G., Historical review: ATP as a neurotransmitter. Trends Pharmacol. Sci. 2006, 27, 166-176.

6. Ashcroft, F. M.; Gribble, F. M., ATP-sensitive $\mathrm{K}^{+}$channels and insulin secretion: their role in health and disease. Diabetologia 1999, 42, 903-919.

7. Bush, K. T.; Keller, S. H.; Nigam, S. K., Genesis and reversal of the ischemic phenotype in epithelial cells. J. Clin. Invest. 2000, 106, 621-626.

8. Lee, S. J.; Jin, Y.; Yoon, H. Y.; Choi, B.-O.; Kim, H. C.; Oh, Y.-K.; Kim, H.-S.; Kim, W.-K., Ciclopirox protects mitochondria from hydrogen peroxide toxicity. Br. J. Pharmacol. 2005, 145, 469-476.

9. Radi, R.; Cassina, A.; Hodara, R.; Quijano, C.; Castro, L., Peroxynitrite reactions and formation in mitochondria. Free Radical Biol. Med. 2002, 33, 1451-1464.

10. Szabó, C.; Ischiropoulos, H.; Radi, R., Peroxynitrite: biochemistry, pathophysiology and development of therapeutics. Nat. Rev. Drug Discovery 2007, 6, 662-680.

11. Valez, V.; Cassina, A.; Batinic-Haberle, I.; Kalyanaraman, B.; Ferrer-Sueta, G.; Radi, R., Peroxynitrite formation in nitric oxideexposed submitochondrial particles: Detection, oxidative damage and catalytic removal by Mn-porphyrins. Arch. Biochem. Biophys. 2013, 529, 45-54.

12. Uribe, P.; Treulen, F.; Boguen, R.; Sánchez, R.; Villegas, J. V., Nitrosative stress by peroxynitrite impairs ATP production in human spermatozoa. Andrologia 2017, 49, e12615.

13. Graves, J. E.; Lewis, S. J.; Kooy, N. W., Role of ATP-sensitive $\mathrm{K}^{+}$-channels in hemodynamic effects of peroxynitrite in anesthetized rats. J. Cardiovasc. Pharmacol. 2005, 46, 653-659.

14. Nilakantan, V.; Liang, H.; Maenpaa, C. J.; Johnson, C. P., Differential patterns of peroxynitrite mediated apoptosis in proximal tubular epithelial cells following ATP depletion recovery. Apoptosis 2008, 13, 621-633.

15. Wang, S.; Chen, L.; Jangili, P.; Sharma, A.; Li, W.; Hou, J.-T.; Qin, C.; Yoon, J.; Kim, J. S., Design and applications of fluorescent detectors for peroxynitrite. Coord. Chem. Rev. 2018, 374, 36-54.

16. Jiao, X.; Li, Y.; Niu, J.; Xie, X.; Wang, X.; Tang, B., Small-molecule fluorescent probes for imaging and detection of reactive oxygen, nitrogen, and sulfur species in biological systems. Anal. Chem. 2018, 90, 533-555.

17. Xiong, J.; Wang, W.; Wang, C.; Zhong, C.; Ruan, R.; Mao, Z.; Liu, Z., Visualizing peroxynitrite in microvessels of the brain with stroke using an engineered highly specific fluorescent probe. ACS Sen. 2020, 5, 3237-3245.

18. Jun, Y. W.; Sarkar, S.; Kim, K. H.; Ahn, K. H., Molecular probes for fluorescent mmaging of ATP in cell and tissue.

ChemPhotoChem 2019, 3, 214-219.

19. Chen, J.; Huang, D.; She, M.; Wang, Z.; Chen, X.; Liu, P.; Zhang, S.; Li, J., Recent progress in fluorescent sensors for druginduced liver injury assessment. ACS Sens. 2021, 6, 628-640.

20. Wu, L.; Liu, J.; Tian, X.; Groleau, R. R.; Bull, S. D.; Li, P.; Tang, B.; James, T. D., Fluorescent probe for the imaging of superoxide and peroxynitrite during drug-induced liver injury. Chem. Sci. 2021, 12, 3921-3928.

21. Cheng, D.; Xu, W.; Gong, X.; Yuan, L.; Zhang, X.-B., Design strategy of fluorescent probes for live drug-induced acute liver injury imaging. Acc. Chem. Res. 2021, 54, 403-415.

22. Al Maruf, A.; Lee, O.; O’Brien, P. J., Modifications of Mitochondrial Function by Toxicants. In Reference Module in Biomedical Sciences, Elsevier: 2014.

23. Masubuchi, Y.; Suda, C.; Horie, T., Involvement of mitochondrial permeability transition in acetaminophen-induced liver injury in mice. J. Hepatol. 2005, 42, 110-116.

24. Wu, L.; Han, H.-H.; Wang, Y.; Tian, X.; Bull, S. D.; Sedgwick, A. C.; He, X.-P.; Huang, C.; James, T. D.; Sessler, J. L., Dual-function fluorescent probe for the detection of peroxynitrite and adenosine triphosphate. ChemRxiv 2019.

25. Wu, Z.; Liu, M.; Liu, Z.; Tian, Y., Real-time imaging and simultaneous quantification of mitochondrial $\mathrm{H}_{2} \mathrm{O}_{2}$ and ATP in neurons with a single two-photon fluorescence-lifetime-based probe. J. Am. Chem. Soc. 2020, 142, 7532-7541.

26. MERCK, https://www.merckmillipore.com/GB/en/product/BioTracker-ATP-LW-Live-Cell-Dye,MM NF-SCT069.

27. Erbas-Cakmak, S.; Kolemen, S.; Sedgwick, A. C.; Gunnlaugsson, T.; James, T. D.; Yoon, J.; Akkaya, E. U., Molecular logic gates: the past, present and future. Chem. Soc. Rev. 2018, 47, 2228-2248.

28. de Silva, P. A.; Gunaratne, N. H. Q.; McCoy, C. P., A molecular photoionic AND gate based on fluorescent signalling. Nature 1993, 364, 42-44. 
29. Wu, L.; Huang, J.; Pu, K.; James, T. D., Dual-locked spectroscopic probes for sensing and therapy. Nat. Rev. Chem. 2021, 5 , 406-421.

30. Sedgwick, A. C.; Han, H.-H.; Gardiner, J. E.; Bull, S. D.; He, X.-P.; James, T. D., The development of a novel AND logic based fluorescence probe for the detection of peroxynitrite and GSH. Chem. Sci. 2018, 9, 3672-3676.

31. Tian, X.; Kumawat, L. K.; Bull, S. D.; Elmes, R. B. P.; Wu, L.; James, T. D., Coumarin-based fluorescent probe for the detection of glutathione and nitroreductase. Tetrahedron 2021, 82, 131890.

32. Wu, L.; Tian, X.; Groleau, R. R.; Wang, J.; Han, H.-H.; Reeksting, S. B.; Sedgwick, A. C.; He, X.-P.; Bull, S. D.; James, T. D., Coumarin-based fluorescent probe for the rapid detection of peroxynitrite 'AND' biological thiols. RSC Adv. 2020, 10, 1349613499.

33. Wu, L.; Gardiner, J. E.; Kumawat, L. K.; Han, H.-H.; Guo, R.; Li, X.; He, X.-P.; Elmes, R. B. P.; Sedgwick, A. C.; Bull, S. D.; James, T. D., Coumarin-based fluorescent 'AND' logic gate probes for the detection of homocysteine and a chosen biological analyte. RSC Adv. 2019, 9, 26425-26428.

34. Wu, L.; Han, H.-H.; Liu, L.; Gardiner, J. E.; Sedgwick, A. C.; Huang, C.; Bull, S. D.; He, X.-P.; James, T. D., ESIPT-based fluorescence probe for the rapid detection of peroxynitrite 'AND' biological thiols. Chem. Commun. 2018, 54, 11336-11339. 35. Fang, Y.; Shi, W.; Hu, Y.; Li, X.; Ma, H., A dual-function fluorescent probe for monitoring the degrees of hypoxia in living cells via the imaging of nitroreductase and adenosine triphosphate. Chem. Commun. 2018, 54, 5454-5457.

36. Liang, X.; Xu, X.; Qiao, D.; Yin, Z.; Shang, L., Dual mechanism of an intramolecular charge transfer (ICT)-FRET-based fluorescent probe for the selective detection of hydrogen peroxide. Chem. Asian J. 2017, 12, 3187-3194.

37. Kirsch, M.; Korth, H.-G.; Wensing, A.; Sustmann, R.; de Groot, H., Product formation and kinetic simulations in the pH range 1-14 account for a free-radical mechanism of peroxynitrite decomposition. Arch. Biochem. Biophys. 2003, 418, 133-150.

38. Phillips, R. C.; George, P.; Rutman, R. J., Thermodynamic data for the hydrolysis of adenosine triphosphate as a function of $\mathrm{pH}, \mathrm{Mg}^{2+}$ ion concentration, and ionic strength. J. Biol. Chem. 1969, 244, 3330-3342.

39. Martin-Romero, F. J.; Gutiérrez-Martin, Y.; Henao, F.; Gutiérrez-Merino, C., Fluorescence measurements of steady state peroxynitrite production upon SIN-1 decomposition: NADH versus dihydrodichlorofluorescein and dihydrorhodamine 123 . $J$. Fluoresc. 2004, 14, 17-23.

40. Hooper, D. C.; Spitsin, S.; Kean, R. B.; Champion, J. M.; Dickson, G. M.; Chaudhry, I.; Koprowski, H., Uric acid, a natural scavenger of peroxynitrite, in experimental allergic encephalomyelitis and multiple sclerosis. Proc. Natl. Acad. Sci. U. S. A. 1998, 95, 675-680.

41. Symersky, J.; Osowski, D.; Walters, D. E.; Mueller, D. M., Oligomycin frames a common drug-binding site in the ATP synthase. Proc. Natl. Acad. Sci. U. S. A. 2012, 109, 13961-13965.

42. Jaeschke, H.; Duan, L.; Nguyen, N. T.; Ramachandran, A., Mitochondrial damage and biogenesis in acetaminophen-induced liver injury. Liver Res. 2019, 3, 150-156.

43. Lee, K. K.; Imaizumi, N.; Chamberland, S. R.; Alder, N. N.; Boelsterli, U. A., Targeting mitochondria with methylene blue protects mice against acetaminophen-induced liver injury. Hepatology 2015, 61, 326-336.

44. Rushworth, G. F.; Megson, I. L., Existing and potential therapeutic uses for N-acetylcysteine: The need for conversion to intracellular glutathione for antioxidant benefits. Pharmacol. Ther. 2014, 141, 150-159.

45. Wang, H.; Zhang, R.; Bridle, K. R.; Jayachandran, A.; Thomas, J. A.; Zhang, W.; Yuan, J.; Xu, Z. P.; Crawford, D. H. G.; Liang, X.; Liu, X.; Roberts, M. S., Two-photon dual imaging platform for in vivo monitoring cellular oxidative stress in liver injury. Sci. Rep. 2017, 7, 45374.

46. Saito, C.; Zwingmann, C.; Jaeschke, H., Novel mechanisms of protection against acetaminophen hepatotoxicity in mice by glutathione and N-acetylcysteine. Hepatology 2010, 51, 246-254.

47. Jaeschke, H.; Xie, Y.; McGill, M. R., Acetaminophen-induced liver injury: from animal models to humans. J. clin. transl. hepatol. 2014, 2, 153-161.

48. Wu, L.; Liu, J.; Li, P.; Tang, B.; James, T. D., Two-photon small-molecule fluorescence-based agents for sensing, imaging, and therapy within biological systems. Chem. Soc. Rev. 2021, 50, 702-734.

49. Hinson, J. A.; Roberts, D. W.; James, L. P., Mechanisms of acetaminophen-induced liver necrosis. In Adverse Drug Reactions, Uetrecht, J., Ed. Springer Berlin Heidelberg: Berlin, Heidelberg, 2010; pp 369-405. 